\title{
Filigrane
}

Écoutes psychanalytiques

\section{Transmettre la psychanalyse : l'Art Brut, un laboratoire pour une première approche de la psychanalyse. Psych'Art Brut, un dispositif pédagogique innovant}

\author{
Pascal Roman, Céline Sayegh et Maud Melchiorre
}

Volume 23, numéro 2, automne 2014

URI : https://id.erudit.org/iderudit/1028925ar

DOI : https://doi.org/10.7202/1028925ar

Aller au sommaire du numéro

Éditeur(s)

Revue Santé mentale au Québec

ISSN

1192-1412 (imprimé)

1911-4656 (numérique)

Découvrir la revue

Citer cet article

Roman, P., Sayegh, C. \& Melchiorre, M. (2014). Transmettre la psychanalyse : l'Art Brut, un laboratoire pour une première approche de la psychanalyse. Psych’Art Brut, un dispositif pédagogique innovant. Filigrane, 23(2), 89-107. https://doi.org/10.7202/1028925ar
Résumé de l'article

Cet article se propose de présenter la démarche pédagogique engagée dans le cadre d'un cours d'Introduction à la psychanalyse donné à l'Université. À partir d'une réflexion sur la faisabilité d'un projet d'enseignement de la psychanalyse, déjà interrogée en son temps par Freud (1919), se dégage une perspective selon laquelle l'approche de la psychanalyse à l'Université (comme théorie, comme méthode, et, dans une moindre mesure comme pratique) gagne à prendre appui sur la rencontre et l'analyse du processus de création. Ainsi, cette démarche pédagogique s'inscrit dans le prolongement de l'intuition freudienne selon laquelle la rencontre avec des oeuvres de création (Freud, 1906) constitue une voie propice pour l'appréhension et l'appropriation des principaux concepts psychanalytiques. Ici, c'est en particulier en appui sur la rencontre d'oeuvres d'Art Brut (avec une collaboration privilégiée avec la Collection de l'Art Brut à Lausanne), que se déploiera l'enseignement. Il s'agit en effet, d'initier les étudiants à repérer, en appui sur un matériel mis à disposition (corpus d'images et de discours sur l'oeuvre ainsi que de films documentaires consacrés à des auteurs d'Art Brut), les indices de la participation d'une des trois principales formes de travail psychique que sont le travail du rêve, le travail du deuil et le travail du jeu au processus de création (à partir des travaux de Anzieu, 1981) et, à partir de là, de leur permettre une première rencontre avec les concepts de base de la psychanalyse. Au-delà de l'énoncé de la démarche didactique qui sous-tend le projet de cet enseignement, l'article présente une illustration clinique à partir d'un auteur se situant à la frontière de l'Art Brut (P. Dereux), ainsi que le synopsis du cours et les modalités d'évaluation de celui-ci. 


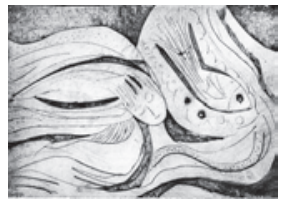

\title{
Transmettre la psychanalyse: I'Art Brut, un laboratoire pour une première approche de la psychanalyse. Psych'Art Brut, un dispositif pédagogique innovant
}

\author{
Pascal Roman, Céline Sayegh \\ et Maud Melchiorre
}

Cet article se propose de présenter la démarche pédagogique engagée dans le cadre d'un cours d'Introduction à la psychanalyse donné à l'Université. À partir d'une réflexion sur la faisabilité d'un projet d'enseignement de la psychanalyse, déjà interrogée en son temps par Freud (1919), se dégage une perspective selon laquelle l'approche de la psychanalyse à l'Université (comme théorie, comme méthode, et, dans une moindre mesure comme pratique) gagne à prendre appui sur la rencontre et l'analyse du processus de création. Ainsi, cette démarche pédagogique s'inscrit dans le prolongement de l'intuition freudienne selon laquelle la rencontre avec des œuvres de création (Freud, 1906) constitue une voie propice pour l'appréhension et l'appropriation des principaux concepts psychanalytiques. Ici, c'est en particulier en appui sur la rencontre d'œuvres d'Art Brut (avec une collaboration privilégiée avec la Collection de l'Art Brut à Lausanne), que se déploiera l'enseignement. Il s'agit en effet, d'initier les étudiants à repérer, en appui sur un matériel mis à disposition (corpus d'images et de discours sur l'œuvre ainsi que de films documentaires consacrés à des auteurs d'Art Brut), les indices de la participation d'une des trois principales formes de travail psychique que sont le travail du rêve, le travail du deuil et le travail du jeu au processus de création (à partir des travaux de Anzieu, 1981) et, à partir de là, de leur permettre une première rencontre avec les concepts de base de la psychanalyse. Au-delà de l'énoncé de la démarche didactique qui sous-tend le projet de cet enseignement, l'article présente une illustration clinique à partir d'un auteur se situant à la frontière de l'Art Brut (P. Dereux), ainsi que le synopsis du cours et les modalités d'évaluation de celui-ci.

a question, soulevée par Freud (1919/1996), de la pertinence d'un enseignement de la psychanalyse à l'Université reste un débat d'actualité. La 
psychanalyse, qui relève tout autant d'un ensemble de savoirs que d'un art de la pratique, pose notamment la question de son intégration et des modalités de sa transmission dans le cadre habituel de l'enseignement académique. L'enseigner peut alors se concevoir également comme un art, qui implique de penser le dispositif pédagogique avec créativité, de manière à engager les étudiants dans un processus actif d'appropriation des concepts théoriques et à les sensibiliser à la pratique psychanalytique au travers d'une démarche personnelle. Dans cette perspective, l'enseignement du cours d'Introduction à la psychanalyse à l'Université de Lausanne, qui présente les principaux fondements théoriques et historiques de la psychanalyse en seconde partie de Bachelor ${ }^{1}$, propose depuis le semestre de printemps 2013 un abord original et vivant de cette matière, dans le cadre du projet d'innovation pédagogique intitulé "Psych'Art Brut», soutenu par le Fonds d'innovation pédagogique (FIP) de l'Université de Lausanne ${ }^{2}$. Dans le cadre de ce projet, il s'agit d'initier les étudiants à la psychanalyse, conçue en tant que théorie mais également en tant que méthode, au travers d'une approche sensible et groupale du processus créateur d'auteurs d'Art Brut. Ainsi, ce dispositif d'innovation pédagogique vise à proposer, via la médiation de la création, une forme d'accès à la psychanalyse et à ses concepts sur le mode du processus d'introjection plutôt que de l'incorporation ${ }^{3}$, en appui sur une invitation à l'expérience du «trouvé-créé».

\section{Le processus de création comme observatoire de la vie psychique: une approche des concepts de la psychanalyse en appui sur le processus de création}

Cette démarche pédagogique se fonde sur la prémisse selon laquelle la rencontre avec des œuvres de création, en particulier celles dites d'Art Brut, constitue une voie propice pour l'appréhension et l'appropriation des principaux concepts psychanalytiques. Plus précisément, il s'agit d'initier les étudiants à repérer, en appui sur un matériel mis à disposition (corpus d'images et de discours sur l'œuvre ainsi que de films documentaires consacrés à des auteurs d'Art Brut), les indices de la participation d'une des trois principales formes de travail psychique que sont le travail du rêve, le travail du deuil et le travail du jeu, au processus de création.

La potentialité heuristique du champ artistique pour l'exploration de la psyché était déjà perçue par l'inventeur de la psychanalyse, qui considérait la création comme une seconde voie royale, avec celle du rêve, pour accéder à l'Inconscient. Celui-ci estimait que: 
[...] ce sont de précieux alliés que les poètes et [que] l'on doit attacher grand prix à leur témoignage, car ils savent toujours une foule de choses entre ciel et terre dont notre sagesse d'école ne peut encore rien rêver. En psychologie, ils sont bien en avance sur nous, hommes du quotidien, parce qu'ils puisent à des sources que nous n'avons pas encore rendues accessibles à la science. (Freud, 1906-1908/2007, 44)

Ainsi, la contribution de la source pulsionnelle archaïque au processus créateur fait de l'œuvre un terrain privilégié d'investigation de l'Inconscient, un témoin des processus primaires engagés dont l'œuvre porte la trace. À l'instar du langage onirique et symptomatique, l'expression artistique autorise une lecture décryptée des procédés intrapsychiques qui, par définition, échappent à toute tentative de saisie. Depuis Freud, nombre d'analystes se sont intéressés au processus de création afin d'enrichir la compréhension psychanalytique du fonctionnement psychique; on peut citer notamment Anzieu qui en livre une modélisation particulièrement élaborée dans son ouvrage «Le corps de l'œuvre: essais psychanalytiques sur le travail créateur» (1981), ouvrage dans lequel il met en évidence la mobilisation du travail du rêve, du deuil et de la création dans le processus créateur.

Les trois axes d'analyse retenus dans le projet Psych'Art Brut présentent une inflexion au regard des propositions d'Anzieu (1981): en effet, la référence au travail du jeu se substitue au travail de la création, dans la mesure où nous considérons qu'il concourt en tant que tel au processus de la création aux côtés du travail du rêve et du travail du deuil, et qu'à ce titre il permet de décrire les processus impliqués dans la création de manière plus spécifique. L'angle d'approche proposé aux étudiants se prête particulièrement à une découverte de la psychanalyse au travers d'une lecture de l'œuvre. En effet, l'attention portée aux trois formes de travail psychique qui concourent au travail de la création - travail du rêve, du deuil et du jeu - permet d'appréhender les processus organisateurs du fonctionnement psychique. Dans cette perspective, la création est envisagée comme travail psychique, c'est-à-dire comme processus de transformation psychique: elle consiste, au travers du processus de symbolisation, en un passage du pulsionnel au registre représentationnel, en une mise en forme et en sens de l'expérience subjective. Ce processus de transformation engage les principes organisateurs de la psyché, témoins du fonctionnement psychique du créateur, dont le travail psychique du rêve, du deuil et du jeu sont à même de rendre compte et dont l'œuvre porte la trace. Autrement dit, la lecture clinique de l'œuvre 
permet d'appréhender la dynamique du fonctionnement psychique de son auteur, dans la mesure où la création et le rapport que l'auteur entretient avec elle témoignent de la nature des conflits psychiques engagés, ainsi que des modalités d'aménagement mobilisées.

\section{Le travail du rêve dans le processus de création}

La contribution du travail psychique du rêve au processus de création a été mise en évidence par Freud, qui considérait:

[...] que sont [...] à l'œuvre dans la production du poète les mécanismes de l'inconscient que nous connaissons à partir du travail de rêve. (Freud, 1906-1908/2007, 41)

En effet, dans la création onirique comme dans la création artistique, une régression topique s'opère, du Conscient vers l'Inconscient, et les procédés inconscients qui se donnent à voir dans le processus créateur sont de même nature que ceux qui sous-tendent la production onirique: condensation, déplacement, déformation. Ces processus primaires, régis par le principe de plaisir et dégagés du jugement rationnel, autorisent l'association libre, le renversement en son contraire, la suspension de la logique spatio-temporelle ainsi que l'absence de la négation et de la contradiction. Au travers de l'activité de symbolisation, le travail psychique de la création mobilise un processus de transformation relevant du travail psychique du rêve: passage d'un contenu latent à un contenu manifeste, mise en forme d'un matériau pulsionnel brut, ébranlement des limites entre réalité interne et externe. En outre, l'illusion créée par le rêve comme par l'œuvre offre un espace de traitement des pulsions et des désirs. La fonction attribuée au rêve, la réalisation hallucinatoire des désirs, s'apparente donc à l'un des enjeux de la création.

\section{Le travail du deuil dans le processus de création}

Le travail psychique du deuil intervient à différents niveaux dans le processus de création. Son enjeu, le traitement du manque et de la perte objectale, consiste en l'établissement de l'objet absent à l'intérieur du Moi. Les objets internes ainsi formés nourrissent l'imaginaire et constituent alors un réservoir de représentations disponibles pour le travail du rêve et, partant, pour la création. La symbolisation à l'œuvre dans l'acte créateur donne donc à voir la qualité de l'intériorisation des objets. Ce processus de transformation est conditionné par l'expérience du deuil: l'absence objectale pousse à 
la fantasmatisation et mobilise le désir de créer des représentations substitutives de l'objet. En effet, il s'agit, pour le créateur, de se présenter à nouveau l'objet manquant, de re-présenter celui-ci différemment, autrement dit, de le rendre présent en lui donnant une nouvelle forme, en le transformant au travers du jeu métaphorique. Sur le plan économique, ce processus implique un retrait de l'investissement à l'égard de l'objet perdu, mouvement qui autorise alors une transformation de cet investissement pulsionnel en une représentation. Ainsi, la symbolisation suppose à la fois une liaison et une séparation objectale: il s'agit de créer un objet dans lequel se retrouvent des attributs de l'objet manquant, tout en maintenant une distance entre celuici et celui-là. Cet écart entre objet à symboliser et objet pour symboliser produit une perte (dont on peut penser le produit comme un reste du travail de symbolisation (Roman et Lempen, 2013)), confrontant le créateur à un travail du deuil, qui réactualise la position dépressive infantile et le deuil fondamental de l'objet primordial. Ainsi, l'œuvre et le lien que le créateur entretient avec elle témoignent des modalités d'élaboration du lien objectal, des mécanismes introjectifs et du processus d'individuation-séparation.

\section{Le travail du jeu dans le processus de création}

En référence au modèle de la transitionnalité conceptualisé par Winnicott (1957/1975), le travail psychique du jeu permet de décrire le champ dans lequel se déploie la symbolisation, au travers du jeu libre chez l'enfant ("play») puis de la création chez l'adulte. Cet espace transitionnel, relevant à la fois de la réalité extérieure et intérieure, du «trouvé-créé», constitue une aire d'illusion qui autorise, en appui sur les images internes construites par le travail du deuil, la mise en scène des conflits psychiques et des situations vécues comme non-vécues. Le travail du jeu contribue au processus de différenciation Moi/non Moi et permet, au travers de mouvements projectifs-introjectifs, l'expérimentation active et l'appropriation subjective des expériences liées notamment à la mise en jeu des limites, à la place du sujet et de l'objet dans le monde, à la séparation et à la perte. Il convoque donc à la fois le travail du rêve et celui du deuil et en condense les enjeux. Au travers du processus de symbolisation, la création témoigne de cette capacité à jouer, à «faire comme si », et à associer suffisamment librement.

\section{Perspectives pédagogiques pour une lecture de l'œuvre}

L'analyse de l'œuvre sous l'angle des différentes formes de travail psychique (travail du rêve, du deuil ou du jeu) permet d'initier les étudiants à 
une démarche psychanalytique de lecture clinique en appui sur des modèles théoriques de la psychanalyse, et les invite à penser en termes de processus et de contenants plutôt qu'en termes de contenus. Loin d'une perspective psycho-biographique ou psychopathologique, l'approche proposée, centrée sur l'identification des mécanismes et des enjeux psychiques qui soustendent le travail créateur, vise à permettre une première compréhension psychanalytique des processus qui fondent l'organisation et le déploiement de la vie psychique. Dans cette optique, l'œuvre se présente comme un support privilégié pour accéder à la dynamique du fonctionnement psychique, qui ne peut être objectivée en tant que telle.

En outre, il s'agit de promouvoir une lecture clinique de l'œuvre, au sens d'une rencontre sensible avec celle-ci, et d'une prise en compte des éprouvés qu'elle suscite. En effet, comme souligné par Anzieu (1981), le travail psychique de transformation engagé dans le processus de création agit sur l'Inconscient du récepteur qui est travaillé par l'œuvre, par ce qui y est symbolisé mais également par ce qui y est déposé en creux, en attente de symbolisation. Ainsi, interpréter une œuvre implique aussi d'élaborer les effets que celle-ci produit sur son propre Inconscient. Cette perspective est particulièrement illustrée par Anzieu dans son ouvrage «Beckett et le psychanalyste» (1992). Dans cette même ligne de pensée, on peut également citer les propos du créateur Duchamp, qui soutient que le récepteur de l'œuvre — le « regardeur» - participe pleinement au processus de création:

Somme toute, l'artiste n'est pas seul à accomplir l'acte de création car le spectateur établit le contact de l'œuvre avec le monde extérieur en déchiffrant et en interprétant ses qualifications profondes et par là ajoute sa propre contribution au processus créatif. (Duchamp, 1957/2013, 28).

Ainsi, à l'instar de la rencontre clinique qui implique une mise au travail des mouvements transféro-contre-transférentiels, cette approche sensible de l'œuvre permet d'introduire les étudiants à la psychanalyse en tant que pratique. Si l'enseignement de la psychanalyse n'a pas vocation a priori de transmettre une formation à la pratique de la psychanalyse, son dispositif pédagogique peut néanmoins être aménagé de sorte à favoriser une première sensibilisation à la méthode et à la pensée psychanalytiques au travers d'une démarche personnelle et vivante, engageant la sensibilité, la créativité et la réflexivité; cette démarche s'appuie sur un implicite, celui de la nature nécessairement projective de l'œuvre de création, œuvre qui porte en elle et 
contribue à une compréhension des processus qui régissent la vie psychique, ainsi qu’y insiste Freud dans ses échanges épistolaires avec Zweig (1995).

\section{Genèse et élaboration d'un dispositif d'innovation pédagogique}

Une collaboration avec la Collection de l'Art Brut de la ville de Lausanne a permis d'inviter les étudiants à découvrir des auteurs d'Art Brut et leurs œuvres selon trois modalités complémentaires : par le biais d'une conférence donnée par L. Peiry, directrice de la recherche et des relations internationales de la Collection de l'Art Brut, par une visite commentée de la Collection, ainsi que par la mise à disposition d'un corpus constitué d'images d'œuvres, d'écrits et de témoignages audiovisuels de dix auteurs d'Art Brut.

L'appui sur un corpus de productions d'Art Brut dans la perspective d'introduire les étudiants à la théorie et à la démarche de la psychanalyse présente un intérêt à plus d'un titre, au-delà du seul motif conjoncturel de l'établissement de la Collection de l'Art Brut dans la ville de Lausanne. En effet, les œuvres d'Art Brut se prêtent spécialement à une exploration dynamique de la psyché et, en particulier, de ses processus archaïques, dans la mesure où elles résultent d'un travail créateur éminemment investi, spontané et intime le plus souvent, affranchi des préoccupations liées à leur réception, aux normes esthétiques en vigueur ou encore au marché de l'art. Par ailleurs, ces formes expressives puissantes, singulières, voire étrangement inquiétantes, facilitent une rencontre sensible de la création de par l'effet saisissant qu'elles provoquent le plus souvent chez le récepteur. En outre, elles se présentent dans une forme de proximité avec ce dernier du fait qu'elles sont produites par des auteurs non professionnels.

Différentes étapes ont présidé à la mise en œuvre de ce projet pédagogique. Une première phase a consisté en un travail d'investigation, aux archives de la Collection de l'Art Brut, afin de sélectionner des auteurs d'Art Brut en vue de la constitution d'un corpus composé d'images, de textes et de documentaires. Ce choix a été effectué à partir de trois critères principaux:

- il s'agissait d'abord de rechercher des productions témoignant, de manière particulièrement explicite, de processus dont les concepts psychanalytiques seraient en mesure de rendre compte;

- il importait ensuite que les sources iconographiques, textuelles et audiovisuelles disponibles soient suffisamment riches;

- enfin, il convenait que les écrits et les documentaires laissent émerger des éléments relatifs au processus créateur des auteurs et à la nature 
de leurs liens avec les œuvres. À cet égard, les données «brutes», témoignages écrits ou audiovisuels des auteurs eux-mêmes, ont été privilégiées. Cette dernière condition n'a pas pu être satisfaite pour l'ensemble des auteurs retenus, dans la mesure où elle requiert que les auteurs disposent d'une suffisante distanciation réflexive vis-à-vis de leur processus de création et qu'ils en livrent un témoignage. Or, comme le souligne Peiry $(1997,11)$ dans son ouvrage, la création dans l'Art Brut se caractérise par «le secret, le silence et la solitude».

Finalement, dix auteurs ont été retenus: Aloïse, Ataa Oko, Eugenio Santoro, Frédéric Bruly Bouabré, Josef Hofer, Marc Moret, Michel Nedjar, Morton Bartlett, Richard Greaves et Sylvain Fusco. Leurs créations se présentent sous des formes variées et originales et embrassent une grande diversité de techniques plastiques: dessins, poèmes, devises, système alphabétique et numérique, sculptures, poupées, photographies, cabanes et installations. À l'issue de la sélection des auteurs, pour chacun d'eux, un corpus a été constitué, composé d'une dizaine d'images (œuvres), d'extraits de textes, de notices biographiques et d'un documentaire dans lequel l'auteur est présenté dans sa pratique de la création et/ou apporte son regard sur son travail créateur et sur ses productions. Ce matériel, issu des archives de la Collection de l'Art Brut, a ensuite été transféré sous forme numérique et déposé sur la plateforme d'apprentissage en ligne Moodle ${ }^{4}$. La dernière étape préparatoire du projet d'innovation pédagogique a consisté à élaborer et à planifier le scénario pédagogique du cours hebdomadaire d'Introduction à la psychanalyse, ainsi que les conditions d'évaluation.

Le premier cours a été consacré à la présentation du projet Psych'Art Brut (visées, modalités, étapes du déroulement pédagogique et conditions et critères d'évaluation), ainsi que des fonctionnalités de la plateforme Moodle et de son contenu (dix corpus relatifs aux auteurs d'Art Brut ainsi qu'une sélection de textes théoriques psychanalytiques ${ }^{5}$ ). En terme de production des étudiants, l'objectif du cours, considéré du point de vue du processus d'appropriation des concepts et de la démarche de la psychanalyse par les étudiants, consiste dans la production, par groupes de quatre à cinq, d'un dossier d'une dizaine de pages rendant compte d'une des trois formes de travail psychique dans le processus de création à partir de l'approche d'un auteur d'Art Brut et de ses productions ${ }^{6}$.

L'évaluation des étudiants à la fin du semestre prend deux formes complémentaires ${ }^{7}$ :

- la production du dossier; 
- un court examen écrit (45 minutes), portant sur la définition et l'illustration de l'un des concepts de la psychanalyse envisagé dans le cours et référé au corpus de texte théorique.

Le cours d'Introduction à la psychanalyse a été rythmé par une alternance entre trois types de contenus ${ }^{8}$ :

- une présentation d'œuvres et de la démarche d'auteurs d'Art Brut;

- un enseignement traditionnel d'éléments historiques et conceptuels de la psychanalyse;

- et la mobilisation d'une démarche réflexive groupale et collaborative9 .

\section{Clinique de la création : repérage des trois formes de travail psychique à partir de l'œuvre de P. Dereux}

Une illustration de la démarche pédagogique mise en ouvre pour soutenir le processus de formation des étudiants à la psychanalyse est proposée à partir de l'œuvre du plasticien P. Dereux. L'artiste français P. Dereux (1918-2001) est un créateur atypique, dont l'œuvre se prête assez bien à la mise en évidence des différentes formes de travail psychique que sont le travail du deuil, le travail du rêve et le travail du jeu. Initialement assistant de J. Dubuffet, qui mieux que lui pourrait rendre compte de la porosité des frontières entre l'Art Brut et l'art dit conventionnel, entre la création dans ses inscriptions les plus archaïques et ses formes les plus inscrites dans la culture?

\section{Quelques repères}

P. Dereux, enseignant dans la région lyonnaise, travaillera pendant plusieurs étés dans la région de Vence pour J. Dubuffet, il sera son «chasseur de papillons", à une époque, dans les années 1950, où ce dernier s'emploie à réaliser des collages en ailes de papillon. Il se définit alors comme «modeste collaboration [...] je dis bien modeste, car j'étais le marmiton qui épluche les légumes pour la cuisine du chef»(Dereux, 1966, 107). Dès les années 1960, P. Dereux se détachera de J. Dubuffet pour engager sa propre production plastique (à défaut d'avoir réussi à écrire, ce qui demeurera pour lui un regret douloureux), production qui se caractérise par l'emploi d'épluchures et d'autres matières végétales. On notera que la référence à la cuisine (la préparation et la cuisson des aliments, le lieu de la création, la référence à la pratique gustative...), qui émaillera l'ensemble de son œuvre, est déjà bien présente dans l'explicitation du rapport entretenu avec J. Dubuffet... lui-même inventeur de l'Art Brut ${ }^{10}$. Il est important de noter d'emblée que 
P. Dereux se définit comme créateur à partir de la mise en forme des restes (alimentaires) et que c'est dans cette marge, doublée d'un repli à l'égard de l'art institutionnel, que l'on peut situer l'appartenance de P. Dereux à l'Art Brut, alors même que l'on ne peut le considérer par ailleurs comme se situant à l'écart de toute inscription et influence culturelles comme le voudraient les critères de définition de l'Art Brut. Outre des œuvres, qu'il produira par centaines des années 1960 à 2000, évoluant d'une production non-figurative (dans laquelle les épluchures constituent une matière plastique qui rehausse la gouache) à une production théâtralisée (que l'on pourrait nommer, en clin d'œil à sa propre nomination des œuvres «théâtre d'épluchures»), la création plastique de P. Dereux se double d'une écriture qui explore et documente son processus de création... ainsi que son lien intime et singulier aux épluchures en particulier ${ }^{11}$.

\section{Les caractéristiques de l'œuvre}

Il convient dans un premier temps de s'arrêter sur la matière de l'œuvre de P. Dereux, matière qui évoluera, on l'a dit, de la peinture (gouache) aux épluchures. Le travail des épluchures occupe une large part de l'œuvre de P. Dereux puisqu'il conditionne la possibilité même de l'œuvre: recueil des épluchures et autres matières végétales (pommes de pin, graines et noyaux divers...), séchage et conservation de celles-ci... La technique de l'œuvre se caractérise par la maîtrise du collage et de la peinture, mais aussi, progressivement, par la fabrication de boîtes qui viendront accueillir les réalisations et autoriseront leur mise en scène. La thématique de l'œuvre s'établit successivement, dans la production de P. Dereux, autour de trois grands axes: d'une part des œuvres non-figuratives, qui semblent dominées par un souci éminemment esthétique et qui s'inscrivent, par leurs titres, dans une perspective que l'on pourrait qualifier d'anthropologique ${ }^{12}$, d'autre part les portraits ${ }^{13}$, qui apparaissent souvent comme des formes de caricatures et, enfin, les «petits théâtres» qui proposent une scénarisation du quotidien ${ }^{14}$.

\section{Le discours sur l'œuvre}

P. Dereux a consacré un certain nombre d'écrits au processus de création qui sous-tend son œuvre, au décours desquels deux affirmations principales émergent:

- l'ouvre s'inscrit dans les contingences du quotidien; elle se trouve indissociable du lien à la nourriture et, partant, de l'expérience de 
satisfaction (orale) qui l'accompagne. Elle ne peut, d'une certaine manière, se soustraire à la satisfaction, au risque d'une dénaturation de la création: il faut rappeler ici que P. Dereux travaille sur la table de la cuisine, avec toutes les limites qu'il reconnaît à cet état de fait, et il attache une importance primordiale à la qualité de l'épluchage qui garantit la qualité de la création (il propose de décerner un «diplôme de bonne éplucheuse», condition préalable au mariage!). Deux extraits du Petit traité des épluchures témoignent de la place de ces contingences:

Pour que j'aie plaisir à coller mes épluchures il faut véritablement qu'elles soient des rebuts, c'est-à-dire que le fruit ou le légume qu'elles contenaient aient servi à l'alimentation. Au fond, les deux joies: manger et coller sont inséparables. Peut-être que le jour où j'achèterai exprès des fruits ou des légumes pour les peaux, sans intention, j'aurai la sensation de faire de l'art et par là je serai moins à l'aise dans ma tentative. (P. Dereux, 1966/1994, 11)

Et, plus loin:

[...] Le dernier melon que nous avons mangé n'était pas franchement bon. Du coup j'ai collé les peaux avec moins de satisfaction. (P. Dereux, 1966/1994, 49)

- l'œuvre répond à un certain nombre de nécessités et de contraintes, tout à la fois attachées à la matière (P. Dereux fait référence aux qualités propres des épluchures, qualités animistes, en évoquant le «pouvoir des peaux») et à la posture mêmes de la création; elle exige de l'auteur une garantie quant au sérieux de sa démarche ainsi qu'une véritable ascèse dans l'art d'accommoder ces restes que sont les épluchures.

\section{L'œuvre, un observatoire des processus psychiques de la création}

L'approche des trois formes de travail psychique (travail du deuil, travail du rêve, travail du jeu) à partir de l'œuvre, invite à interroger ce qui, tout à la fois du point de vue de l'œuvre (de ses modalités techniques et expressives, de la rencontre de sa singularité) et du discours sur l'œuvre, autorise une lecture clinique de l'œuvre. La rencontre des œuvres de création de P. Dereux ouvre ainsi sur une triple perspective: 
- autour du travail du deuil, en lien avec la matière brute de la création et le processus de transformation qui en fonde la nécessité: l'œuvre peut-elle être considérée comme une mise en forme de l'informe?

- autour du travail du rêve, avec l'investissement de la peau et la limite qu'elle instaure: l'œuvre ne constitue-t-elle pas une forme d'enveloppe du rêve?

- autour du travail du jeu, par le jeu avec la matière et le détournement figuratif qu'il autorise: l'œuvre ressortit-elle d'un espace du trouvé-créé?

Dans la perspective d'une mise en forme de l'informe, le travail du deuil peut être repéré à partir d'un enjeu central dans l'œuvre de P. Dereux, celui du traitement des expériences de perte. En effet, l'ensemble de l'œuvre se trouve mobilisé vers le projet de traiter les expériences de perte au travers du traitement des restes, restes que l'on peut bien sûr référer aux peaux issues des épluchures, peaux que l'on peut entendre comme une métaphore des restes de l'enfance. Ce traitement passe par un certain nombre de figures qui concourent au travail du deuil:

- la récupération de la matière brute de l'œuvre et la perpétuation, sur le mode du déplacement, du plaisir oral qu'elle autorise dans l'œuvre;

- l'évitement de l'expérience de la perte inhérente au fait de conserver, de coller, de transformer autorise un réinvestissement pulsionnel de l'objet perdu au travers de la satisfaction orale et/ou anale;

- la lutte contre la destruction (disparition) qui se traduit tout spécialement dans la préoccupation de l'auteur pour la conservation des œuvres.

Considérant l'œuvre de P. Dereux du point de vue de la construction d'une enveloppe de rêve, le travail du rêve peut être identifié au travers de la mise à l'épreuve, par la médiation du visuel, du lien entre réalité interne et réalité externe. On peut relever à cet égard:

- l'expression onirique des premières œuvres: Danchin (1995) parle à cet égard de "pointillisme hypnotique»;

- l'inscription imaginaire et/ou fantasmatique des œuvres qui contribuent à la création de nouvelles figurations, que l'on pourrait comprendre comme la création d'un nouveau monde, quête d'un ancrage anthropologique de l'œuvre qui participe d'une vision universalisante des représentations proposées, au-delà des frontières des catégories habituelles;

- la mise en scène du regard et les "petits théâtres ", qui mobilisent la dynamique entre voir et être $v u$ : l'auteur invite au rêve et à l'échappée 
auquel celui-ci autorise, et inscrit, dans l'œuvre même, la place du regardeur.

Enfin, le travail du jeu peut être envisagé à partir du détournement figuratif (par déplacement, à nouveau) auquel appelle l'œuvre, détournement qui prend appui sur une mise à l'épreuve de l'objet:

- la trace de la destructivité et le potentiel créateur qui en émerge, apparaissent centraux dans le processus de création: si l'épluchure du fruit témoigne de l'effraction de l'enveloppe de l'objet, celle-ci se trouve secondairement suturée dans le collage, restaurée dans son intégrité;

- la figure du trouvé/créé s'inscrit comme fondement dans l'œuvre, et transparaît également dans le discours sur l'œuvre, tout particulièrement lorsque P. Dereux fait référence aux qualités animiques des peaux;

- par ailleurs, la mise en scène théâtrale de son œuvre (les "petits théâtres») témoigne d'une forme d'aboutissement du travail du jeu, dans la mesure où l'objet, restauré dans son intégrité, se trouve à même de soutenir une potentialité d'adresse, inscrit dans l'intersubjectivité.

Le repérage de la contribution des trois formes de travail psychique au processus de création met en évidence le fait que le potentiel créateur s'appuie sur une poursuite du projet libidinal (ici lié à l'oralité): pour P. Dereux, le plaisir de coller se substitue au plaisir de manger, permettant à l'auteur d'éviter l'expérience de la perte et de trouver, ainsi qu'il le dit lui-même, une forme de répit et de réassurance.

\section{Apports du dispositif et perspectives}

Au travers des différents éléments de son dispositif pédagogique, le projet Psych'Art Brut s'inscrit dans la thématique du Fonds d'innovation pédagogique 2013 de l'Université de Lausanne, soit la promotion de la pratique collaborative afin de potentialiser les apprentissages. En effet, par le biais de l'instauration de forums de discussion et de séances de travail collectif, le dispositif pédagogique permet d'encadrer et de valoriser la dynamique coopérative. En outre, celle-ci s'accorde de manière significative à la nature même du projet d'introduire les étudiants à la psychanalyse au travers d'une approche réflexive et créative, dans la mesure où la confrontation de son propre regard à celui des membres de son groupe et d'autres groupes favorise, par un effet de synergie, la stimulation et le déploiement fructueux des idées. Le groupe peut alors se constituer comme espace de rêverie diurne et de pensées collectives. L'objectif pédagogique de cette démarche groupale 
réside donc dans la volonté de favoriser l'appropriation des concepts psychanalytiques. Au-delà de cette visée, l'expérimentation du travail en équipe est formatrice pour les étudiants en vue de leur future pratique professionnelle. Dans cette perspective, ces derniers étaient régulièrement invités à donner leur appréciation de l'apport du travail intra et intergroupal.

Cette approche pédagogique cherche également à renforcer l'implication personnelle des étudiants dans leur processus d'apprentissage en promouvant une rencontre sensible avec les œuvres d'Art Brut. En effet, l'engagement des affects, par la médiation de la création, dans la découverte de la psychanalyse, favorise davantage un investissement personnel de la part des étudiants que ne peut le faire un enseignement traditionnel. Le groupe se présente comme réceptacle et transformateur des émergences contre-transférentielles liées à la rencontre de l'œuvre, dans une fonction de "conteneur» au sens de Kaës (1979/1990). En outre, le maniement réflexif et créatif des concepts psychanalytiques en appui sur un corpus d'œuvres facilite l'intégration de ceux-ci, car il permet aux étudiants de les aborder sous une forme actualisée et de manière vivante. Si le cours d'Introduction à la psychanalyse s'appuyait déjà sur des productions artistiques à titre illustratif, il s'agissait jusque-là d'une présentation faite aux étudiants plutôt que d'une expérimentation par ces derniers. Cette nouvelle modalité pédagogique les engage à une prise de position active qui ne peut que renforcer le processus d'appropriation.

Un autre intérêt de ce projet réside dans l'opportunité d'une collaboration avec la Collection de l'Art Brut, dans un rapport de réciprocité. En effet, la Collection de l'Art Brut a permis la constitution d'un corpus à partir de ses sources iconographiques, textuelles et audiovisuelles, et a accepté d'ouvrir ses portes aux étudiants, permettant ainsi à l'enseignement académique de bénéficier d'un lien avec une institution culturelle de la cité. De son côté, l'université offre une lecture originale du processus créateur et des œuvres d'Art Brut en proposant à la Collection de l'Art Brut de diffuser sur son site le meilleur dossier parmi les travaux des étudiants, sélectionné par un comité réunissant les deux institutions.

Par ailleurs, la reconduction de cette nouvelle approche pédagogique est assurée par le maintien d'un lien vivant avec la Collection de l'Art Brut et par la constitution du corpus de départ, susceptible d'être enrichi dans le cadre de futurs travaux de master ou de doctorat.

Enfin, la présente initiative pédagogique s'intègre à une tradition vivante d'enseignement de la psychanalyse s'appuyant sur la portée heuristique du 
processus de création. Ainsi, l'Université de Lausanne propose, dans les plans d'étude de master en psychologie et en lettres, un cours d'Art et psychanalyse dispensé par T. Diamantis, Privat-docent. En France, en Belgique et en Grande-Bretagne notamment, certaines universités adoptent une démarche similaire: la référence au réseau international interuniversitaire Clinique de la création ${ }^{15}$, espace de recherche qui s'intéresse au processus de création comme voie d'exploration des processus psychiques aussi bien au niveau métapsychologique que clinique, est à ce titre incontournable.

En fonction des opportunités locales, on pourrait imaginer que des dispositifs pédagogiques similaires soient développés en appui sur des institutions culturelles qui seraient en mesure de présenter des créations en marge du circuit artistique normatif ${ }^{16}$.

Pascal Roman pascal.roman@unil.ch

Céline Sayegh

Maud Melchiorre

Annexe 1 - Synopsis du cours semestriel d'Introduction à la
psychanalyse (semestriel, 2h)
\begin{tabular}{|c|l|}
\hline Séances & Contenu \\
\hline 1 & $\begin{array}{l}\text { Argument du cours et organisation (objectifs, scénario pédagogique, } \\
\text { planning, validation) }\end{array}$ \\
\cline { 2 - 3 } & $\begin{array}{l}\text { Présentation du corpus d'œuvres d'Art Brut et des fonctionnalités de } \\
\text { Moodle }\end{array}$ \\
\hline 2 & L'Art Brut, ses origines et ses auteurs (conférence de L. Peiry) \\
\hline 3 & $\begin{array}{l}\text { Les différentes formes de travail psychique : travail du rêve, travail du } \\
\text { deuil et travail du jeu }\end{array}$ \\
\hline 4 & Une brève histoire de la psychanalyse (1) \\
\cline { 2 - 3 } & $\begin{array}{l}\text { Séance de travail en groupement par auteur (1) : choix de l'angle } \\
\text { d'approche (travail psychique du rêve, du deuil ou du jeu), } \\
\text { constitution des groupes et répartition des rôles au sein de chaque } \\
\text { groupe }\end{array}$ \\
\hline 5 & Visite de la Collection de l'Art Brut \\
\hline 6 & La perspective économique du fonctionnement psychique \\
\hline 7 & Les perspectives topique et dynamique du fonctionnement psychique \\
\hline
\end{tabular}




\begin{tabular}{|c|c|}
\hline Séances & Contenu \\
\hline \multirow[t]{2}{*}{8} & Une brève histoire de la psychanalyse (2 ) \\
\hline & $\begin{array}{l}\text { Séance de travail intra-groupe (2): identification des apports de } \\
\text { chacun des membres du groupe }\end{array}$ \\
\hline 9 & $\begin{array}{l}\text { Clinique du travail du rêve, du deuil et du jeu dans le processus de } \\
\text { création de P. Dereux (illustration) }\end{array}$ \\
\hline 10 & Les étapes du développement pulsionnel \\
\hline 11 & La construction de la transitionnalité \\
\hline 12 & $\begin{array}{l}\text { Psychanalyse et création : désir de créer, besoin de créer, contrainte à } \\
\text { créer }\end{array}$ \\
\hline 13 & Psychanalyse et psychopathologie \\
\hline \multirow[t]{2}{*}{14} & Épreuve de contrôle continu \\
\hline & $\begin{array}{l}\text { Séance de travail inter-groupe (3) : présentation de la version } \\
\text { provisoire du dossier à d'autres groupes centrés sur la même forme de } \\
\text { travail psychique }\end{array}$ \\
\hline
\end{tabular}

\section{Annexe 2 - Contenu du dossier}

Le dossier doit comporter:

- une partie individuelle soulignant, en appui sur des éléments du corpus, la participation du travail du rêve, du deuil ou du jeu dans la création de l'auteur choisi;

- une partie collective proposant, pour l'auteur retenu et en référence aux concepts présentés en cours et aux textes psychanalytiques mis à disposition sur Moodle, une compréhension des enjeux du travail psychique choisi dans le processus créateur en question;

- une note d'appréciation de la démarche collaborative.

La méthodologie a été exposée au travers d'un découpage en trois étapes: lecture sensible de l'œuvre (accueil des impressions et des éprouvés suscités par l'œuvre), mise en perspective avec les aspects théoriques du travail psychique, construction d'une compréhension clinique des enjeux soulevés par l'œuvre.

\section{Annexe 3 - Dynamique du cours et démarche collaborative}

Les étudiants sont introduits à l'Art Brut au travers d'une conférence, donnée par L. Peiry, sur les origines de l'Art Brut et ses auteurs, au travers d'une visite commentée de la Collection de l'Art Brut invitant à se saisir de la rencontre sensible avec les œuvres exposées et à faire l'expérience du 
«regardeur», ainsi qu'au travers d'une exploration personnelle du corpus mis à disposition en vue du choix d'un auteur parmi les dix proposés. En outre, les étudiants bénéficient d'un accès libre à la Collection de l'Art Brut tout au long du semestre.

La part classique de l'enseignement consiste en une présentation des différentes formes de travail psychique, des perspectives économique, topique et dynamique du fonctionnement psychique, des fondements historiques et des développements de la psychanalyse, des étapes du développement pulsionnel ainsi que de la construction de l'objet en référence au modèle de la transitionnalité. Des écrits psychanalytiques relatifs aux trois formes de travail psychique ont été déposés sur la plateforme Moodle. Par ailleurs, une lecture clinique du travail du rêve, du deuil et du jeu dans le processus créateur de Philippe Dereux, auteur d'Art Brut ${ }^{17}$, a été présentée aux étudiants à titre d'illustration.

La dynamique collaborative a été soutenue par l'instauration, à deux reprises, de forums de discussion sur Moodle et par l'organisation de trois séances de travail collectif durant le cours:

- dans un premier temps, dix forums ont été ouverts afin de permettre aux étudiants ayant fait le choix de travailler à partir du même auteur de partager leur réflexion autour de l'identification des trois formes de travail psychique dans le processus créateur de l'auteur retenu. Une première séance de travail, entre étudiants travaillant sur le même auteur, est ensuite aménagée afin de permettre à chacun de déterminer la forme de travail psychique (travail psychique du rêve, du deuil ou du jeu) à partir de laquelle envisager l'approche de l'œuvre, puis de procéder à la constitution finale des groupes (en fonction du choix commun de l'auteur et du travail psychique) et à la répartition des rôles de chaque membre, chacun travaillant sur des sources spécifiques du corpus (extraits de textes et/ou de documentaires), secondairement mis en perspective avec les œuvres et le processus de création de l'auteur. Dès lors, d'autres forums de discussion ont été ouverts sur Moodle, offrant la possibilité aux étudiants ayant choisi le même point de vue (travail du rêve, du deuil ou du jeu) de nourrir leur réflexion au travers de leurs échanges.

- une deuxième séance de travail de groupe est consacrée à la présentation par chacun des membres du groupe de l'avancée de son travail devant les membres de son groupe, afin de permettre une identification des apports de chacun au travail et le dégagement de pistes en vue d'une élaboration collective, 
- la troisième séance de travail, réunissant les groupes centrés sur la même forme de travail psychique dans le processus créateur à partir d'auteurs différents, vise à favoriser un enrichissement du travail par l'apport du regard d'autres groupes. A l'issue de chacune de ces séances, les étudiants étaient invités à réfléchir à l'apport du travail coopératif.

\section{Notes}

1. Équivalent de la licence en France, du baccalauréat en Belgique ou au Québec: le cours peut être suivi en deuxième ou troisième année de Bachelor.

2. Le Fonds d'innovation pédagogique de l'Université de Lausanne est une initiative qui vise l'amélioration des conditions d'apprentissage des étudiants en promouvant et en soutenant des projets novateurs en matière d'enseignement.

3. Au sens où l'entendent respectivement S. Ferenczi et S. Freud, et au travers de la dialectique qu'en proposera plus tard M. Klein (1934).

4. Ce dispositif, auquel recourt l'Université de Lausanne, permet de mettre à la disposition des étudiants, sur une interface numérique dédiée, des documents ainsi que diverses fonctionnalités, liées notamment à l'organisation des cours et à la démarche collaborative.

5. Neuf textes ont été mis à la disposition des étudiants sur la plateforme numérique «Des cinq phases du travail créateur et de leurs inscriptions dans l'œuvre (Anzieu, 1981); «Le délire et les rêves dans la 'Gradiva'de W. Jensen » (Freud, 1907/2007); «Le Moïse de Michel-Ange» (Freud, 1914/2005); «Les situations d'angoisse de l'enfant et leur reflet dans une œuvre d'art et dans l'élan créateur» (Klein, 1921-1945/1976); «Le stade du miroir comme formateur de la fonction du Je» (Lacan, 1949); «Le rôle de l'illusion dans la formation du symbole» (Milner, 1952); «Figures du négatif et «expérience» créatrice» (Roman, 2007); «Désir de créer, besoin de créer, contrainte à créer, capacité de créer» (Roussillon, 1998); «La créativité et ses origines» (Winnicott, 1957/1975).

6. Voir annexe 2 .

7. La note obtenue à l'épreuve de contrôle continu vaut pour $40 \%$ de la note, celle obtenue pour la réalisation du dossier pour $60 \%$.

8. Voir en annexe 1 le synopsis du cours.

9. L'annexe 3 présente la dynamique du cours et les éléments contribuant à la mise en œuvre de la démarche collaborative.

10. Inventeur de l'Art Brut... à tout le moins au sens où c'est J. Dubuffet qui, le premier, prendra une initiative d'ampleur, tout à la fois en termes de recherche et de collection d'œuvres d'auteurs marginaux et en termes d'organisation de ce corpus dans le cadre de la fondation d'une institution consacrée à ces œuvres, en l'occurrence la Collection de l'Art Brut à Lausanne, à défaut d'avoir pu l'installer à Paris ou ailleurs en France dans des conditions satisfaisantes (Peiry, 1997, 172).

11. On peut se référer en particulier à: Petit traité des épluchures (1966 / 1994), et à: Sagesse des épluchures (2001).

12. Comme en témoigne la "Stèle funéraire pour un chef indien», la «Monade» ou le «Médaillon couché».

13. «Une forte femme» ou «Shéhérazade» par exemple.

14. On peut penser aux «Foules du stade» ou aux «Justiciers». 
15. Ce réseau est conduit par Anne Brun à l'Université Lumière - Lyon 2 et par Céline Masson à l'Université Paris 7 - Diderot, où se réunit le groupe de recherches Pandora. C'est dans ce cadre de collaboration que le colloque «Matière brute, matière à symboliser» a été organisé en 2010 à l'Université de Lausanne, en partenariat entre Pandora, la Collection de l'Art Brut et l'Unil.

16. On peut penser, par exemple à Paris, aux institutions de la Halle Saint Pierre ou de la Maison Rouge, ou à Bruxelles à Art \& Marges Musée.

17. Les œuvres de P. Dereux peuvent être retrouvées sur le site de la galerie Chave (galeriechave. com/artiste-philippe-dereux. html); elles sont également évoquées sous le terme de Neuve Invention sur le site de la Collection de l'Art Brut (http://www.artbrut.ch/ fr/21074/neuve-invention).

\section{Références}

Anzieu, D., 1981, Le corps de l'œuvre: essais psychanalytiques sur le travail créateur, Paris, Gallimard.

Anzieu, D., 1992, Beckett et le psychanalyste, Paris, Mentha.

Danchin, L., 1995, Art Brut et compagnie: la face cachée de l'art contemporain, Paris, La Différence.

Dereux, P., 1966/1994, Petit traité des épluchures, Paris, Juilliard.

Dereux, P., 2001, Sagesse des épluchures, Marseille, L'œuf sauvage.

Duchamp, M., 1957/2013, Duchamp du signe, Paris, Flammarion.

Freud, S., 1907/2007, Le délire et les rêves dans la «Gradiva» de W. Jensen, in Euvres complètes VIII, 39-126, Paris, Presses universitaires de France.

Freud, S., 1914/2005, Le Moïse de Michel-Ange. in Euvres complètes XII, 127-160, Paris, Presses universitaires de France.

Freud, S., 1919/1996, Doit-on enseigner la psychanalyse à l'université?, in CEuvres complètes XV, 109-114, Paris, Presses universitaires de France.

Freud, S., Zweig, S., 1995, Correspondance, Paris, Payot et Rivages.

Kaës, R., 1979/1990, Introduction à l'analyse transitionnelle, in Kaës, R. et al., Crise, rupture et dépassement, 1-83, Paris, Dunod.

Klein, M., 1921-1945/1976, Les situations d'angoisse de l'enfant et leur reflet dans une œuvre d'art et dans l'élan créateur, in Essais de psychanalyse, 254-262, Paris, Payot.

Klein, M., 1934/1976, Contribution à l'étude de la psychogenèse des états maniaco-dépressifs, in Essais de psychanalyse, 311-340, Paris, Payot.

Lacan, J., 1949/1966, Le stade du miroir comme formateur de la fonction du Je, in Écrits I, 89-97, Paris, Seuil.

Milner, M., 1952/1998, Le rôle de l'illusion dans la formation du symbole, in Chouvier, B., éd., Matières à symbolisation, 29-59, Lausanne, Champ-Vallon.

Peiry, L., 1997, L'Art Brut, Paris, Flammarion.

Roman, P., 2007, Figures du négatif et «expérience» créatrice, in Brun, A., Talpin, J.-M., éds, Cliniques de la création, 117-140, Paris, De Boeck.

Roman, P., Lempen, O., 2013, Traumatisme et restes à symboliser: une contrainte à créer?, in Dumet, N., éd., De la maladie à la création, Toulouse, Erès (L'ailleurs du corps), 93-108.

Roussillon, R., 1998, Désir de créer, besoin de créer, contrainte à créer, capacité de créer, in Chouvier, B., et al., Symbolisation et processus de création: sens de l'intime et travail de l'universel dans l'art et la psychanalyse, 158-171, Paris, Dunod.

Winnicott, D. W., 1957/1975, La créativité et ses origines, in Jeu et réalité, 127-161, Paris, Gallimard, coll. Folio/essais. 